\title{
Electric Relaxation Processes in Lipid-Bilayers after Exposure to Weak Magnetic Pulses
}

\author{
Alexander Pazur \\ Botanisches Institut der Universität München, Menzingerstr. 67, D-80638 München, \\ Germany.
}

Fax. 089-17861-185. E-mail: pazur@botanik.biologie.uni-muenchen.de

Z. Naturforsch. 56c, 831-837 (2001); received March 29/April 27, 2001

Black Lipid Membrane, Magnetic Field Effect, Quantum Effects

Biological effects of weak magnetic fields are widespread, but poorly understood. Besides magnetic particles, which have been shown to be involved in only few cases, membranes are discussed as the site of perception. However, the mechanism is unknown. We have subjected pure lipid membranes to weak magnetic pulses, and found, that their electric properties are modified.

Black lipid membranes were prepared from purified asolectin on a teflon septum separating electrically the two chambers of a teflon cuvette, using the technique of Mueller et al. (1962). Single magnetic pulses were applied for $10 \mu \mathrm{s}$, whose intensity could be varied from 0 to $100 \mathrm{G}(0$ to $10 \mathrm{mT})$ at the membrane. Directly after the pulse decay, the conductance of the bilayers was scanned with 10 periods of a $1 \mathrm{kHz}$ triangle alternating voltage (eg. a measurement time window of $10 \mathrm{~ms}$ ). Frequency spectra of the bilayer current rose by a frequency dependent factor $\leq 2$ in a broad region around $80 \mathrm{kHz}$, when the amplitude of the preceding magnetic pulse was increased from 0 to $100 \mathrm{G}$. The data show, that weak magnetic fields can significantly change the electrical conductance of lipid films. The relaxation of electrons in a two-dimensional quantum state ("quantum hollow") will be discussed as a possible origin of these effects.

\section{Introduction}

Magnetic fields and non ionizing electromagnetic radiation in the frequency range from extremely low (ELF) to ultra high frequencies (UHF) have gained an increasing public interest in the last two decades, in view of their potential physiological effects on humans (WHO, 1984; WHO, 1987), animals, plants and microorganisms. Naturally generated components of such fields are the result of complex interactions between electrical processes in the atmosphere, charged solar and interstellar particles, and the earth's magnetic field (Schumann, 1952; Heavner and Sentman, 1996). They change generally only on long timescales, with one exception beeing electric bursts in the upper atmosphere as a result of rising solar activity, which recur with a period of approximately 11 years. A correlation was shown between higher solar activities and ischemic heart diseases (Stoupel et al., 1999).

By contrast, the environmental contribution by man-made electromagnetic fields rose rapidly during the last decades as a consequence of progress in electrification, communications engineering, and medical diagnostics (see review by Preece $e t$ al., 2000). Since little has been known on the physiological significance of sub thermic magnetic field strengths, they became a focus of interest in medical research. (Burch et al., 1999) investigated modulations of melatonin excretion as a result of combinations of weak natural and man-made electromagnetic fields. An overwiev about similar investigations on plants was compiled by Phirke $e t$ al. (1996).

Three physical mechanisms are currently considered as origins of biological effects of magnetic fields at sub thermal energies: ferromagnetism, chemical reactions with singlet-triplet mixing, and cyclotron resonances of ions. Magnetic field receptors based on the presence of ferromagnetic particles in monocellular organisms and in the the nervous systems of some vertebrates are increasingly understood (Torres et al., 1986; Lohmann and Johnsen, 2000). Likewise ferrit particles could act in combination with the visual system of animals in some cases as a "compass mechanism" (Deutschlander et al.; 1999; Wiltschko et al., 2000). 
A magnetic field effect involving the singlet-triplet-state mixing is known for photosynthetic reaction centers (Ogrodnik et al.,1982; Vrieze and Hoff, 1990; Waliszewski et al., 1999). Working at field strengths below $1000 \mathrm{G}(0.1 \mathrm{~T})$, this "radical pair mechanism" is able to modulate the triplet yield of pre-reduced reaction centers to $100 \%$, compared experiments without an external magnetic field. Weaver et al. (2000) reported about combination effects with ferromagnetical receptors.

Magnetically induced cyclotron resonances of ions in aqueous solutions controling the yields of biochemical reactions are considered by Liboff (1997). In addition to this, recent studies about biological effects of low magnetic fields suggest phenomena, which are based on ordered matter in the nanometer size scale, so-called "nano-structures". Biological membranes could belong to these structures (Galt et al., 1993; Volpe et al., 1998). Here results are reported, which show, that "naked“ lipid bilayers change their conductivity, when magnetic pulses of relatively low intensity are applied.

\section{Materials and Methods}

\section{Preparation}

Asolectin (soybean-lecithin, Fluka) with a primary L- $\alpha$-phosphatidylcholine (PC) content of approx. $30 \%$ was purified and enriched to $75 \%$ PC by silicagel-thin layer chromatography after (Klenk et al., 1961) and identification after (Nichols, 1964). A stock solution was prepared with $2 \%$ w/v lipid in $n$-decane for use.

\section{Apparatus}

Black lipid membranes (BLMs) were prepared after (Mueller et al., 1962; Tien and Diana, 1968; Matews and van Holde 1990). The lipid solution was dispersed with a soft paintbrush (da Vinci Cosmotop-spin 5580) over an aperture with a diameter of $1.5 \mathrm{~mm}$ in a teflon film with a thickness of $0.1 \mathrm{~mm}$, which seperated the two $25 \mathrm{ml}$ compartments of a teflon chamber. The chambers were filled with a $100 \mathrm{mM} \mathrm{MgCl}$ solution in ultrafiltrated, doubly deionized water, thermostated at $20 \pm 0.5^{\circ} \mathrm{C}$. The membranes showed a good long time stability. The electric connections from the electrolyte to the electronic equipment were provided by two graphite electrodes.

The chamber was mounted on the top of an Helmholtz coil (diameter $17 \mathrm{~cm}$, inductivity 3.4 $\mathrm{mH}$, see Fig. 1), no ferromagnetic components were located in a range of $0.7 \mathrm{~m}$ around the apparatus. The electromagnetic properties of the coil were calibrated with a semiconductor-based Hall magnetometer. Signal coordination and data sampling was done by a PC with a home built signalprocessor and transient recorder for high sample rates with 12 bit resolution. Magnetic pulses with $10 \mu$ s duration and intensities of $0-100 \mathrm{G}(0-10$ $\mathrm{mT}$, pulse strength defined as $\Phi=B \cdot t$ of $0-100$ $\mathrm{mT} \cdot \mu \mathrm{s}$, see Fig. 2 , top) were generated by a timing logic and coupled to the coil with a wide band power amplifier. Pulse amplitudes were controled by the measurement software, monitored by inductive coupling to a detector coil $(0.29 \mathrm{mH})$ winded on the core of the exciter coil. They were visualized with an oscilloscope (Nicolet 4094 C). A field inhomogeneity of less than $2 \%$ was determined in the range of $2 \mathrm{~cm}$ around the BLM locus by a home built semiconductor Hall detector.

Subsequent to the magnetic pulse, a train of 10 triangle pulses (duration $1 \mathrm{~ms}$ each) with a peak to peak amplitude of $\pm 100 \mathrm{mV}$ was applied to

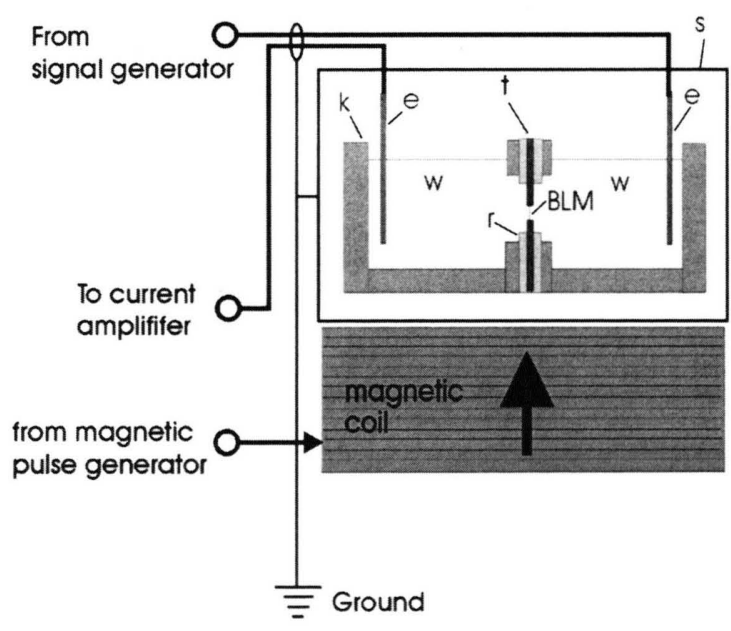

Fig. 1. Schematic diagram of the cuvette with the BLM samples and the measuring system (components are not drawn to scale): e, electrodes; $k$, cuvette; w, water phase $\mathrm{t}$, teflon septum; r rubber seal; s, electrical shielding. The large arrow gives the direction of the magnetic field. 


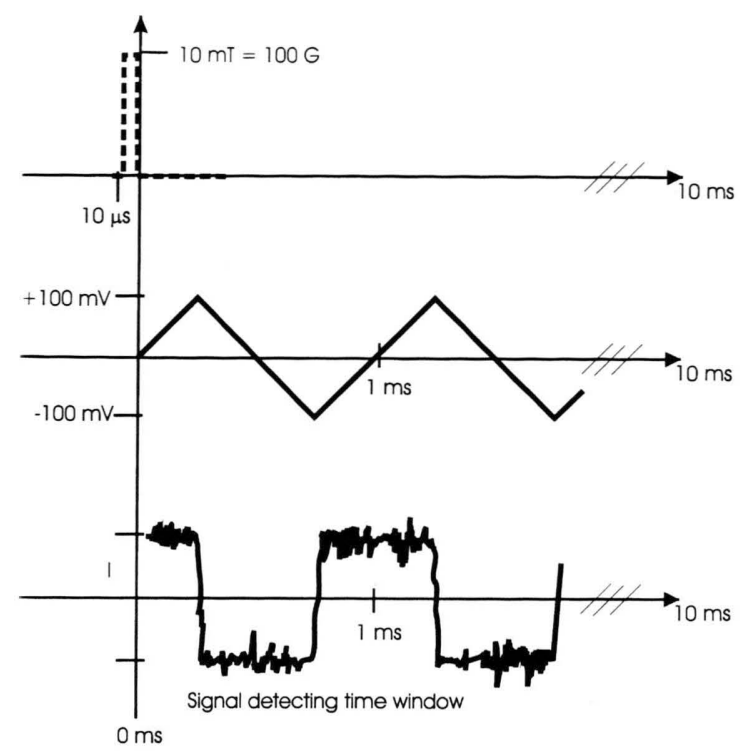

Fig. 2. Magnetic (top) and electric signals (center) applied to the BLMs, and the response (bottom).

Top: Magnetic pulse sent immediately before the beginning of measuring the BLM currents (width not drawn to scale).

Center: Triangular scanning voltage with $100 \mathrm{mV}$ amplitude and $1 \mathrm{~ms}$ period length.

Bottom: Schematic course of a typical BLM current for the applied triangle voltage. It has basically the shape of a square-signal (the derivative of a triangle signal) due to the capacitive properties of the BLM. This signal was used for the further frequency analysis by FFT.

one of the bath electrodes, (see Fig. 2, center) while the current through the BLM was detected by the other electrode. The amplifier input impedance was $100 \Omega$. After preamplification and sampling with a rate of $256 \mathrm{kHz}$, the frequency spectrum was obtained bei fast Fourier transformation (FFT) in the range of 2 to $128 \mathrm{kHz}$, with a resolution of $0.25 \mathrm{kHz}$. 8 such spectra were internally averaged, then the magnetic pulse height was increased in $1 \mathrm{G}$ steps from 0 to $100 \mathrm{G}$ for one complete experiment.

\section{Results}

The experiments described were carried out with pure lipid membranes. A lipid film was spread over the hole of a teflon septum, and the membrane formation monitored by its electrical resistance. Approx. $10 \mathrm{~min}$. after application the resistance reached a steady state level with a deviation of $\leq 0.1 \%$ of the total current amplitude, and the measurement could be started. It should be tried to measure the current response of a ,naked" BLM on the applied alternating triangle voltage in combination with the magnetic pulses with varying intensities. Therefore it was needed to build up a BLM under stable environmental conditions with a lifetime as long as possible. Most preparations showed reproducible signals for at least $20 \mathrm{~min}$., which is long enough to carry out 34 repetitive experiments with a single BLM. A total of 40 experiments were performed, 20 with, and 20 without applying magnetic pulses. The current induced by the triangle wave, looked at first sight like a square-wave signal with some noise, as expected for a probe with mostly capacitive electric properties (see Fig. 2, bottom). Only even harmonic frequencies should appear for a pure square-wave signal, but these dominated only the lower frequencies. At higher frequencies in contrast to the signal of an electric capacitor, the FFT spectrum showed extensive activities of the even $(2,4,6, \ldots)$ higher harmonics. Upon increasing the magnetic pulse strength $\Phi$, the amplitude of the FFT-spectra rose in a broad band centered about $70-100 \mathrm{kHz}$. Several experiments were carried out to ensure that this behavior is characteristic of the BLM and not an artefact of the apparatus. The magnetic field response could never be seen, if the BLM state was not yet reached, when using an open septum without any lipid film, when inserting an electronic capacitor instead of the BLM device into the circuit, or when the septum was closed with an adhesive tape (thickness approx. $50 \mu \mathrm{m}$ ).

The intensities of FFT spectra decrease tenfold and more for higher harmonics in most cases. Therefore, the spectra in the presence of a magnetic field were normalized to those obtained without a magnetic field. Fig. 3 shows the (dimensionless) amplitude ratios $I_{100} / I_{0}$ of the spectra obtained at maximum strength of the magnetic pulses $(100 \mathrm{mT} \cdot \mu \mathrm{s})$, over those obtained in the absence of any magnetic pulse. The BLMs show a maximum ratio of $2.036( \pm 0.030)$ at $77.5 \mathrm{kHz}$. The total averaged currents investigated over the entire frequency domain were $8.43( \pm 0.27) \mathrm{pA} / \mathrm{Hz}$ without any magnetic pulse and $11.77( \pm 0.49) \mathrm{pA} /$ $\mathrm{Hz}$ at maximum magnetic pulse $(100 \mathrm{mT} \cdot \mu \mathrm{s})$ for all comparable experiments.

For further analysis the magnetic pulse strength dependence of the BLM currents were evaluated 


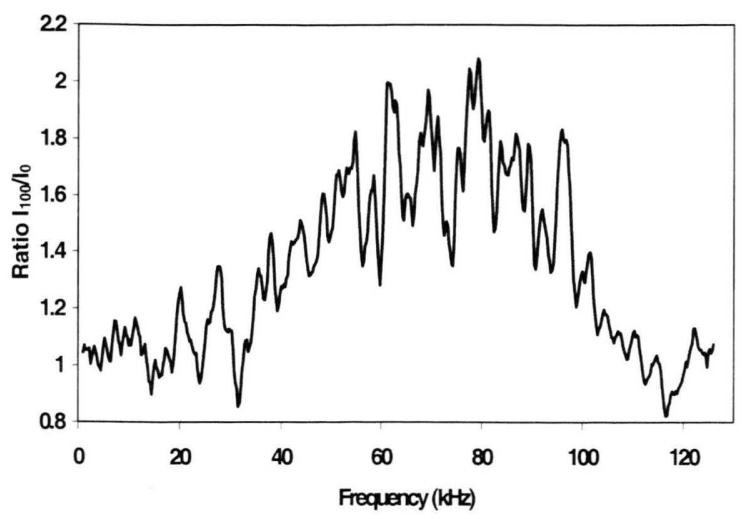

Fig. 3. Amplitude ratios of the spectra for full magnetic pulse intensity to those at zero intensity. Signficant ratio changes appeared in a broad band centered around 80 $\mathrm{kHz}$. The ratio $\mathbf{I}_{100} / \mathbf{I}_{0}$ is the dimensionless quotient of the corresponding spectra.

for the three discrete frequencies $f=40,80$, and $120 \mathrm{kHz}$, which mark approximately the borders and the maximum of the observed spectral band. The total current in a frequency window of $250 \mathrm{~Hz}$ (the resolution of the FFT-spectra) was normalized by its bandwidth, and expressed as $\mathrm{pA} / \mathrm{Hz}$. These currents rose with increasing magnetic pulse intensity. In Fig. 4 they are already compensated by subtraction of the values for zero magnetic field, which amount to $6.1 \mathrm{pA} / \mathrm{Hz}$ at $40 \mathrm{kHz}, 4.4$ $\mathrm{pA} / \mathrm{Hz}$ at $80 \mathrm{kHz}$, and $4.5 \mathrm{pA}$ at $120 \mathrm{kHz}$. Table I summarizes the data.

The data indicate, that although the BLM currents increase more or less monotonously with the magnetic pulse strength, the deviations from the

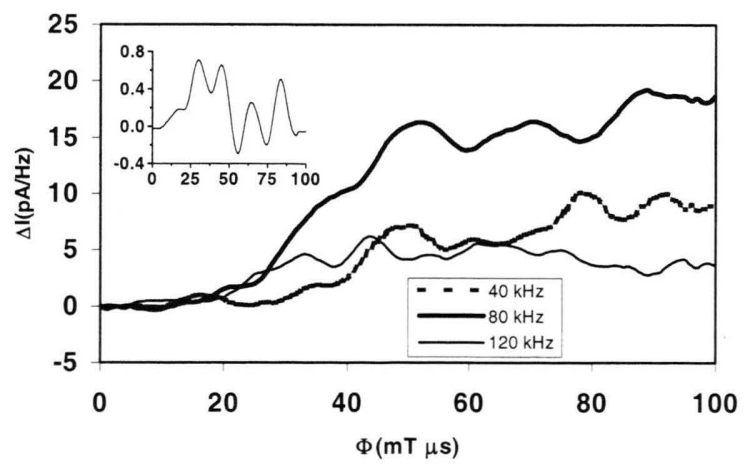

Fig. 4. Current increase frequency bands 40, 80, and 120 $\mathrm{kHz}$, in the dependence of the intensity of the magnetic pulse, applied directly before measuring . The inserted graph shows the first derivative of the data for $80 \mathrm{kHz}$. linear gradient suggest a structured signal, rather than a simple noise phenomenon. The average periods of these variations were calculated by derivation analysis, and a quotient $\mathrm{dB} / \mathrm{df}$ was obtained. It amounts to $4.15 \cdot 10^{-8} \mathrm{~T} / \mathrm{Hz}$ for all equivalent experiments. In summary there is therefore a twofold effect of weak magnetic fields on the ,naked" BLM. The BLM currents increase with the amplitude of the magnetic pulse. This increase is frequency specific, and at each frequency roughly proportional to magnetic pulse strength. In addition, small but significant modulations are observed by the magnetic field.

\section{Discussion}

Two models shall be explored below as explanations for the low magnetic field effects below the range of thermal energy exchange, which could be able to generate the non linear, superimposed periodic current increase on a linearly rising field strength. Both models assume domains of coupled electron spins over distances which are large compared with the BLM thickness, or more precisely: the thickness of the lipid-water transitron zones on both sides of the BLM).

For the observed magnetic field effects, an origin from ferromagnetic substances can be excluded, they were absent. Experiments were not done under a protection gas atmosphere (like Argon). Therefore the formation and involvement of oxigen radicals is possible in principle, similar effects are suggested by (Colic et al., 1999). But on the one hand they should prevent the reproduction of data on the same BLM, because they involve non reversible chemical reactions effecting on long time scales, on the other hand the electric force produced by these radicals would need to overcome the inner membrane potential barrier, in order to cause a directed current through the BLM, which is unlikely. Similar considerations will apply for ionic cyclotron resonance effects, in the absence of ion channels.

Quantum mechanics describe a free electron not as a single point, but as a standing wave in a finite volume. Depending on the energy, this wave may have a relatively large spatial coherence. Most famous is the "tunnel effect", basing on this knowledge, but many other physical description tools are built on it. Tunneling at extreme low energies 
Table I. Magnetic field effects on the conductivity of BLMs: The current increase at the BLMs within a $250 \mathrm{~Hz}$ band centered at 40,80 , and $120 \mathrm{kHz}$. Data are averaged from 20 independent repetitions of each experiment, standard deviations therefore are shown for total increase of the magnetic pulse strength from 0 to $100 \mathrm{mT} \cdot \mu \mathrm{s} . I_{\mathrm{f}}$ $(\mathrm{pA} / \mathrm{Hz})$ is the current (in $\mathrm{pA}$ ) normalized to a (theoretical) bandwidth of $1 \mathrm{~Hz} . \Delta \boldsymbol{\Phi}(\mathrm{mT} \cdot \mu \mathrm{s})$ represents the product of the field strength (in $\mathrm{mT}$ ) and duration (in $\cdot \mu \mathrm{s}$ ) of the magnetic pulse (integral). The unit $\mathrm{pA} /(\mathrm{Hz} \cdot \mathrm{mT} \cdot \mu \mathrm{s})$ indicates the gradient $I_{\mathrm{f}} / \Delta \boldsymbol{\Phi}$ of the linear regression.

\begin{tabular}{|c|c|c|c|c|}
\hline \multirow[t]{2}{*}{ Parameter } & \multirow[t]{2}{*}{ Physical unit } & \multicolumn{3}{|c|}{ Frequency range } \\
\hline & & $f=40 \mathrm{kHz}$ & $f=80 \mathrm{kHz}$ & $f=120 \mathrm{kHz}$ \\
\hline $\begin{array}{l}\text { Current increase for } \\
(0-100 \cdot \mathrm{mT} \cdot \mu \mathrm{s})\end{array}$ & $I_{\mathrm{f}}(\mathrm{pA} / \mathrm{Hz})$ & $\begin{array}{l}10.22 \\
\pm 1.66\end{array}$ & $\begin{array}{l}19.62 \\
\pm 0.86\end{array}$ & $\begin{array}{l}6.21 \\
\pm 0.45\end{array}$ \\
\hline $\begin{array}{l}\text { Proportionality factor of a } \\
\text { linear regression }\end{array}$ & $\mathrm{pA} /(\mathrm{Hz} \cdot \mathrm{mT} \cdot \mu \mathrm{s})$ & +0.115 & +0.226 & +0.039 \\
\hline $\begin{array}{l}\text { Full frequency band current } \\
\text { increase } \\
(0-100 \cdot \mathrm{mT} \cdot \mu \mathrm{s})\end{array}$ & $I_{\mathrm{f}}(\mathrm{pA} / \mathrm{Hz})$ & & $1049 \pm 98$ & \\
\hline${ }^{1}$ Superimposed periodicity & $\Delta \boldsymbol{\Phi}(\mathrm{mT} \cdot \mu \mathrm{s})$ & \multicolumn{3}{|c|}{$32.2 \pm 2.83($ at $77.5 \mathrm{kHz})$} \\
\hline
\end{tabular}

${ }^{1}$ The widths of the periodic variations superimposed to the linear current increase were obtained by derivation analysis. They were used to calculate a gyromagnetic magneton (see text).

( $\mu \mathrm{eV}$ range) in acetly halides is described by (Jahnke et al., 1995). Further (Binhi et al., 2000) consider quantum-interference effects for ions in biological tissue, resulting in an unusual "amplitude windows" behavior for the conduction of low currents. We will try to estimate the possible distances of charge interactions of uniform electron assemblies on a membrane firstly by their gyromagnetic moment, possibly caused by spin coupling, and secondly by the coherence lengths, predicted by the electronic wave function.

\section{Calculating a magneton (gyromagnetic moment)}

The magneton $\beta$ for an electron (Bohr magneton) is defined as:

$$
\boldsymbol{\beta}=\frac{e \cdot h}{4 \pi \cdot m_{e}}=9.274 \cdot 10^{-24} \mathrm{~J} / \mathrm{T} \text {. }
$$

(Electron charge $e=1.6 \cdot 10^{-19}$ As, Planck constant $h=6.626 \cdot 10^{-34}$ $\mathrm{J} / \mathrm{s}$, electron mass $m_{\mathrm{e}}=9.109 \cdot 10^{-31} \mathrm{~kg}, \pi=3.141$ )

Generally it can be writen as $\boldsymbol{\beta}=\frac{h \cdot f}{B}$

( $f=$ frequency, $B=$ magnetic field strength).

Using the $\Delta \Phi / f$ ratios from the above experiments, we obtain magnetons of $1.59 \cdot 10^{-26} \mathrm{~J} / \mathrm{T}$. This magneton is smaller by a factor of 581 compared to the Bohr magneton. The first-order explanation for this decrease would be an indication, that charge coupled clusters with $581 \mathrm{~m}_{\mathrm{e}}$, equal to number of electrons, are responsible for the magnetic field effect.
Considering the average charge density of the conductive electrons, calculated by the BLM surface (approx. $\mathbf{A}_{\mathrm{B}}=7.8 \cdot 10^{-7} \mathrm{~m}^{2}$ ) and the BLM currents, we get a diameter of approx. $103 \mathrm{~nm}$ for these electron spin coupled regions.

\section{Assuming a coherent electron wave function:}

One criterion for magnetic field effects as a result of long distance electronic coupling should be an uniform wavefunction over this distance. Such wavelenghts $\lambda$ for electrons or other particles can be calculated by the equation of de Broglie (here for electrons):

$$
\lambda=\frac{h}{\sqrt{2 \cdot m_{e} \cdot U}}
$$

Here $U$ is the potential of the electron (for other units see eqation (2)), and $e \cdot U$ presents the energy in "electron Volt" $\left(1 \mathrm{eV}=1.6 \cdot 10^{-19} \mathrm{~J}\right)$. Calculations, based on the experimental data for the energy dissipation of the magnetic field for a free, mobile electron can only be made by the assumption, that the expected relaxation times are large, compared with the exitation time $(10 \mu \mathrm{s})$, which is not fully met by the spectral maxima around $80 \mathrm{kHz}$ (a period time of $12.5 \mu \mathrm{s}$ ) averaged over the measure time window of 10 scanning periods with $\left(t_{\mathrm{p}}=1 \mathrm{~ms}\right.$ duration each) after magnetic pulse. Otherwise, no frequency dependent or independent magnetic field effect will be seen without applying the electric scanning, so that there should be a real interaction between the 
magnetic field and the charge transfer from the BLM surface to the water phase, driven by external electric force. So we can write for the time independent unit area energy density $\mathbf{E}_{\mathrm{A}}$ (unit $\mathrm{J} / \mathrm{m}^{2}$ ) for a scanning period $t_{\mathrm{p}}$, resulting from the effective full frequency band currents (see Table I), renormalized by the frequency band resolution factor $d f=250 \mathrm{~Hz}$ :

$$
\mathbf{E}_{A}=\frac{\Phi \cdot I_{f} \cdot d f}{t_{p}}
$$

For the previousely assumed BLM surface $\mathbf{A}_{\mathrm{B}}$ we can insert an enery term in Equ. (3):

$$
\lambda=\frac{h}{\sqrt{2 m_{e} \cdot E_{A} \cdot A_{B}}}
$$

Binhi V. N. and Goldman R. J. (2000), Ion-protein dissociation predicts "windows" in electric field-induced wound-cell proliferation. Biochim. Biophys. Acta 1474, 147-156.

Burch J. B., Reif J. S. and Yost M. G. (1999), Geomagnetic disturbances are associated with reduced nocturnal excretion of a melatonin metabolite in humans. Neurosci Lett. 266, 209-212.

Colic M. and Morse D. (1999), The elusive mechanism of the magnetic 'memory' of water. Colloids Surf. 154, $167-174$.

Conley C. C. (1969), Effects of near-zero magnetic fields upon biological systems. In: Biological Effects of Magnetic Fields. Vol. 2 (M. F. Barnothy, Ed.) Plenum Press, New York, pp. 29-31.

Deutschlander M. E., Phillips J. B. and Borland S. C. (1999), The case for light-dependent magnetic orientation in animals. J. Exp. Biol. 202, 891-908.

Galt S., Sandblom J., Hamnerius Y., Hojevik P., Saalman E. and Norden B. (1993), Experimental search for combined AC and DC magnetic field effects on ion channels. Bioelectromagnetics 14, 315-327.

Heavner M. J. and Sentman D. D. (1996), Ionospheric height correction analysis of long-term, multi-station Schumann resonance observations. Proceedings of the 10th Intern. Conf. on Atmospheric Electricity.

Hirota N., Nakagawa J. and Kitazawa K. (1999), Effects of a magnetic field on the germination of plants. J. Appl. Phys. 85, 5717-5719.

Jahnke T. K., Müller-Warmuth W. and Bennati M. (1995), Anomalous proton relaxation, rotational tunnelling and barriers to methyl group rotation in solid acetyl halides, Solid State Nucl. Magn. Reson. 4, $153-161$.

Klenk E. and Gielen W. (1961), cited by: Kurt Randerath, Dünnschicht-Chromatographie (1965), Vol. 2, pp. 171-172, Verlag Chemie, Weinheim.

Liboff A. R. (1997), Electric-field ion cyclotron resonance. Bioelectromagnetics 18, 85-87.

Lohmann K. J. and Johnsen S. (2000), The neurobiology of magnetoreception in vertebrate animals. Trends Neurosci. 23, 153-159.

Matews C. K. and van Holde K. E. (1990), Biochemistry, p. 306, Benjamin/Cummings Publ. Comp., Redwood City.
For the full frequency band currents from Table 1 we get a wavelength $\lambda=108 \mathrm{~nm}$. That means, the results of both calculations correspond within approximately five per cent.

\section{Acknowledgements}

The author thanks H. Scheer (München) for scientific care about many years, and H. P. Braun (München) for frequent discussions, and for providing mechanical equipment for bilayer experiments.

Mueller P., Rudin P. D. O, Tien H. Ti. and Wescott W. C. (1962), Reconstitution of cell membrane structure in vitro and its transformation into an excitable system. Nature, 194, 979-980.

Nichols B. W. (1964), Separation of plant phospholipids and glycolipids, in: New Biochemical Separations (A. T. Janes and I. J. Morris, Eds.). Van Nostrand, London, pp. 23-39.

Ogrodnik A., Krüger H. W., Orthuber H., Haberkorn R. and Michel-Beyerle M. E. (1982), Recombination dynamics in bacterial photosynthetic reaction centers. Biophys J. 39, 91-99.

Phirke P. S., Kubde A. B. and Umbarkar S. P. (1996), The influence of magnetic field on plant growth. Seed Sci. Technol. 24, 375-392.

Preece A. W., Hand J. W., Clarke R. N. and Stewart A. (2000), Power frequency electromagnetic fields and health. Where's the evidence? Phys. Med. Biol. 45, $139-154$.

Schumann W. O.(1952), Über die strahlungslosen Eigenschwingungen einer leitenden Kugel, die von einer Luftschicht und einer Ionosphärenhülle umgeben ist. Z. Naturforsch. 7a, 149.

Stoupel E., Petrauskiene J., Abramson E., Kalediene R., Israelovich P. and Sulkes J. (1999), Realtionship between deaths from stroke and ischemic heart disease - environmental implications, J. Basic Clin. Physiol. Pharmacol. 10, 135-145.

Tien H. T. and Diana A.L (1968), Bimolecular lipid membranes: a review and a summary of some recent studies. Chem. Phys. Lipids 2, 55-101.

Torres de Araujo F. F., Pires M. A., Frankel R. B. and Bicudo C. E. M. (1986), Magnetite and magnetotaxis in algae, Biophys. J. 50, 375-378.

Volpe P., Parasassi T., Esposito C., Ravagnan G., Giusti A. M., Pasquarelli A. and Eremenko T. (1998), Cell membrane lipid molecular dynamics in a solenoid versus a magnetically shielded room. Bioelectromagnetics 19, 107-111.

Vrieze J. and Hoff A. J., Exciton band mixing in Rhodopseudomonas viridis (1990) in: Reaction Centers of Photosynthetic Bacteria Vol. 6, (M. E. Michel-Beyerle Ed.). Springer Publ., Berlin, pp. 409-421. 
Waliszewski P., Skwarek R., Jeromin L. and Manikowski, H. (1999), On the mitochondrial aspect of reactive oxygen species action in external magnetic fields. J. Photochem. Photobiol. 52b, 137-140.

Weaver J. C., Vaughan T. E. and Astumian R. D. (2000), Biological sensing of small field differences by magnetically sensitive chemical reactions. Nature 405, 707-709.

WHO (1984), Natural background and man-made ELF fields. In: Environmental Health Criteria, Vol. 35, Ex- tremely Low Frequency Fields. World Health Organization, Geneva, pp. 32-50.

WHO (1987), Biological interactions. In: Environmental Health Criteria, Vol. 69, Magnetic Fields. World Health Organization, Geneva, pp. 15-25.

Wiltschko R., Wiltschko W. and Munro U. (2000), Lightdependent magnetoreception in birds: the effect of intensity of 565-nm green light. Naturwissenschaften 87, 366-369. 